\title{
Review Article \\ Rare Earth Doped Silica Optical Fibre Sensors for Dosimetry in Medical and Technical Applications
}

\author{
N. Chiodini, ${ }^{1}$ A. Vedda, ${ }^{1}$ and I. Veronese ${ }^{2}$ \\ ${ }^{1}$ Dipartimento di Scienza dei Materiali, Università di Milano-Bicocca, Via Cozzi 55, 20125 Milano, Italy \\ ${ }^{2}$ Dipartimento di Fisica, Università degli Studi di Milano, Via Celoria 16, 20133 Milano, Italy
}

Correspondence should be addressed to N. Chiodini; norberto.chiodini@mater.unimib.it

Received 22 April 2014; Accepted 23 September 2014; Published 14 October 2014

Academic Editor: Adam S. Wyatt

Copyright (c) 2014 N. Chiodini et al. This is an open access article distributed under the Creative Commons Attribution License, which permits unrestricted use, distribution, and reproduction in any medium, provided the original work is properly cited.

\begin{abstract}
Radioluminescence optical fibre sensors are gaining importance since these devices are promising in several applications like high energy physics, particle tracking, real-time monitoring of radiation beams, and radioactive waste. Silica optical fibres play an important role thanks to their high radiation hardness. Moreover, rare earths may be incorporated to optimise the scintillation properties (emission spectrum, decay time) according to the particular application. This makes doped silica optical fibres a very versatile tool for the detection of ionizing radiation in many contexts. Among the fields of application of optical fibre sensors, radiation therapy represents a driving force for the research and development of new devices. In this review the recent progresses in the development of rare earth doped silica fibres for dosimetry in the medical field are described. After a general description of advantages and challenges for the use of optical fibre based dosimeter during radiation therapy treatment and diagnostic irradiations, the features of the incorporation of rare earths in the silica matrix in order to prepare radioluminescent optical fibre sensors are presented and discussed. In the last part of this paper, recent results obtained by using cerium, europium, and ytterbium doped silica optical fibres in radiation therapy applications are reviewed.
\end{abstract}

\section{Introduction}

An optical fibre based dosimeter basically consists of a small scintillator coupled to a passive fibre of suitable length for remote signal transport to an optical detector (e.g., photomultiplier, photodiode, etc.). Such configuration has several advantages in radiation dosimetry applications. Indeed, the small volume of the detector makes the radiation field perturbation negligible, leading to high spatial resolution and point dose evaluations. These systems may enable a real-time measurement of the dose, providing a direct feedback to the medical physician during a radiation therapy (RT) treatment. In vivo measurements on patients take also advantage by the lack of any electrical supply.

Furthermore, provided that a suitable scintillator material is used, an optical fibre based dosimeter is characterized by high sensitivity and reproducibility, independence of sensitivity upon accumulated dose, independence of the response from the environmental conditions, linearity of the response over a wide range of doses/dose rates, high radiation hardness, and nontoxicity for medical applications.

However, for an effective use of these systems as dosimeters in RT, the stem effect, that is, the spurious luminescence that is produced as consequence of the irradiation of the passive optical fibre, still remains one of the main challenges to face. Indeed, if the spurious signal is not properly subtracted from the total scintillation signal, the dosimetric properties of the system may be drastically impaired.

The possible mechanisms causing the stem effect during the irradiation of optical fibre based dosimeters are fluorescence and phosphorescence phenomena, as well as Cerenkov light [1]. Fluorescence may occur in the light guide due to the intrinsic scintillation properties of the material constituting the optical fibre (typically silica but often different polymeric materials have also been used). Phosphorescence is caused by charge trapping and detrapping phenomena, an effect of the intrinsic defects in fibre material [2]. Finally, Cerenkov radiation is generated when the charged particles (i.e., electrons for 


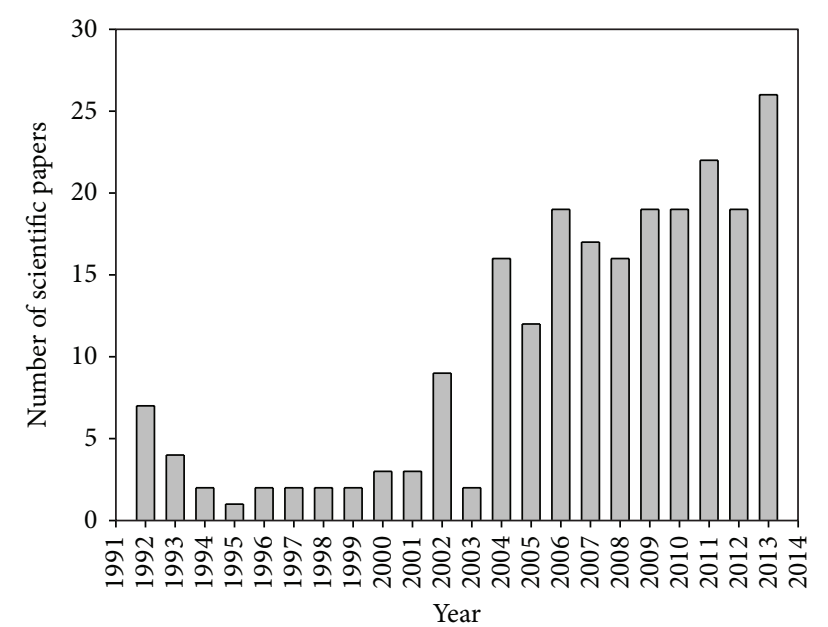

FIGURE 1: Temporal distribution of the manuscripts about the use of optical fibre based dosimeters in radiation therapy available in literature (from Scopus).

conventional RT) pass through the medium at a speed greater than the speed of light in the medium itself [3].

The first studies aimed at exploiting the properties of optical fibre based scintillation dosimeters in radiation therapy date back to the early 90s. Beddar and colleagues [4] investigated the physical characteristics of water-equivalent plastic scintillation detectors for high energy beam dosimetry and tried to face the problem of the stem effect through the use of a second reference fibre [5]. Indeed, the system consisted of two optical fibre light guides running parallel to each other, one optically coupled to a plastic scintillator and the other used to subtract the background signal due to the radiation-induced light generated in the optical fibre.

The interest for such types of dosimetric systems remained rather limited during the following years, as attested by the relative few research papers available in the literature in that period (Figure 1). As examples, de Boer and colleagues [6] performed spectral measurement of radiation-induced light in plastic scintillator dosimeters and investigated the feasibility of optical filtering for the stem effect subtraction. Flühs and colleagues [7] developed dosimeters based on plastic scintillators for a variety of applications in radiation therapy and, in particular, employed such systems for eye plaque dosimetric treatment optimization. Létourneau and colleagues [8] studied the properties of a miniature scintillating detector, suitable for small field radiation dosimetry, using a configuration similar to that of Beddar [4] for facing the limitation due to the stem effect.

Over the last decade, significant improvements were made in radiation therapy technologies. In fact, the new medical linear accelerators or dedicated technologies are now able to irradiate the planned target volumes and avoid adjacent organs at risk using intensity-modulated fields [9, 10]. Moreover, increased use is being made of radiosurgery for small cranial lesions and stereotactic body radiation therapy for small body targets. These progresses called for a simultaneous improvement of detectors and dosimeters for the maintenance of a satisfactory quality assurance programme of these systems. Furthermore, the possibility to perform prompt checks of the delivered dose through in vivo dosimetry studies becomes extremely important for RT treatments characterised by a high dose per single fraction [11].

Optical fibre based radioluminescence dosimeters are a promising option for these purposes and therefore the interest for these types of detectors is rapidly increasing, as well as the number of papers on this topic available in the literature (Figure 1). In fact, various groups are performing research on these types of detectors, considering different organic and inorganic scintillating materials $[12,13]$. In addition to the choice of the scintillating material, the recent research has addressed the implementation of an efficient procedure for removing the stem effect contribution. Various methods were proposed in literature to distinguish the scintillation signal from the spurious one. The above mentioned first approach based on the use of a second reference fibre to separately measure the spurious signal [4] was proved to give reliable results in various tests under controlled conditions, but it showed some weaknesses with modern treatment modalities [12]. Moreover, although simple from a theoretical point of view, this approach has the disadvantage to increase the size of the detector, worsening therefore the spatial resolution, keyelement in case of stereotactic radiation field monitoring.

A second method, initially proposed by Clift and colleagues [14] and then successfully implemented by Andersen and colleagues [15], is based on the temporal discrimination between the scintillating signal of interest and the Cerenkov light. This approach can be efficiently used with rather slow inorganic scintillators, characterised by long scintillation decay times, provided that the spurious signal is mainly due to the Cerenkov effect and not to other slower fluorescent phenomena occurring in the optical fibre material.

A third method for discriminating the dosimetric luminescence signal from the spurious one is based on optical separation of their emissions. After the first paper of de Boer and colleagues [6], chromatic stem effect removal approaches, making use of interference or dichroic filters, were proposed in more recent literature, especially for plastic scintillators [16, 17]. Guillot and colleagues [18] showed that, using this method, a high level of accuracy in correcting measurements for the effect of Cerenkov radiation can be achieved. The main limit of this approach is the elaborate and critical calibration procedure initially required [12]. As far as the authors know, the only system available in the market (i.e., Exradin W1 Scintillator, Standard Imaging) is based on this discrimination method.

An alternative approach aimed at preventing Cerenkov light was followed by Lambert and colleagues [19] through a suitable design of the radioluminescent dosimeter, that is, by including a rigid air core light guide between the scintillator and the optical fiber. The weak aspect of this system is the poor mechanical flexibility which may introduce difficulties in in vivo dosimetry studies.

In addition to the external radiation therapy by means of innovative accelerators, a rapid diffusion of High-DoseRate (HDR) brachytherapy units occurred in the last few 
years. In this context, optical fibre based dosimeters proved to be of great interest for in vivo dosimetry application, thanks to their real-time response and the possibility of dose evaluation to inner organs and tissues close to the planned target volumes [20-24].

Our research has mainly addressed the development and application of doped silica optical fibres. Indeed, silica optical fibres are characterised by high radiation hardness and, therefore, they are promising for dosimetry applications in high radiation environments. Moreover, various rare earths may be considered as dopants in the silica matrix in order to obtain scintillators with different RL properties, in terms of both emission spectrum and decay time. This makes doped silica optical fibres an interesting versatile tool for ionizing radiation monitoring in a broad range of applications.

The road toward the production of suitable optical fibre based dosimeters was characterized by three main phases:

(1) preparation of silica glass bulk samples by sol-gel method and their preliminary optical and physical characterization,

(2) production of preliminary optical fibre sensors by "powder in tube" method,

(3) standardisation of the procedure for optical fibre sensors preparation by drawing a monolithic rod produced entirely by sol-gel method.

\section{Experimental Methods and Results}

2.1. Preparation and Characterisation of Silica Glass Bulk Samples. Silica glasses with rare earth (RE) molar ppm concentration $(\mathrm{mol} \mathrm{ppm}=($ moles of $\mathrm{RE} /($ moles of $\mathrm{RE}+$ moles of $\mathrm{SiO} 2)) \times 10^{6}$ ) in the range $0-5000 \mathrm{ppm}$ were prepared by the sol-gel method.

Tetraethyl orthosilicate (TEOS, Aldrich, 99.999\%), RE$\left(\mathrm{NO}_{3}\right)_{3} \cdot 6 \mathrm{H}_{2} \mathrm{O}$ (Aldrich, 99.99\%), [RE = Ce, Eu, Gd, Tb, Yb, etc.] were used as precursors.

TEOS $(2.0 \mathrm{~mL}$ ) was mixed with ethanol (HPLC grade reagent) and with suitable volume of $0.1 \mathrm{M}$ ethanol solutions of $\mathrm{Ce}\left(\mathrm{NO}_{3}\right)_{3} \cdot 6 \mathrm{H}_{2} \mathrm{O}$ or other $\mathrm{RE}$, according to the glass composition. The volume of pure ethanol introduced in the SOL solution depended on the amount of RE solution, as the total volume was $8.0 \mathrm{~mL}$. Finally, $1.20 \mathrm{~mL}$ of water (Merck analytical grade) was added under stirring $\left(\mathrm{H}_{2} \mathrm{O}\right.$ :TEOS molar ratio 7.4 ).

The resulting clear solutions were sealed in polypropylene containers (5 $\mathrm{cm}$ in diameter) and stored in a thermostatic chamber at $35^{\circ} \mathrm{C}$. Gelation occurred in 10-20 days. Afterwards, samples were aged for 2 days and, subsequently, small holes were produced in the container covers in order to induce slow drying of the alcogel. Drying of the alcogels was reached in about $1-2$ weeks at $35^{\circ} \mathrm{C}$, yielding transparent xerogels. After a couple of months of aging, densification of xerogels to glasses was carried out through a sintering procedure up to $1050^{\circ} \mathrm{C}$.

Specifically, samples were heated under an oxygen stream up to $450^{\circ} \mathrm{C}$ (heating rate $6^{\circ} \mathrm{C} / \mathrm{h}$ ) and maintained at this temperature for $24 \mathrm{~h}$; then the samples were more slowly

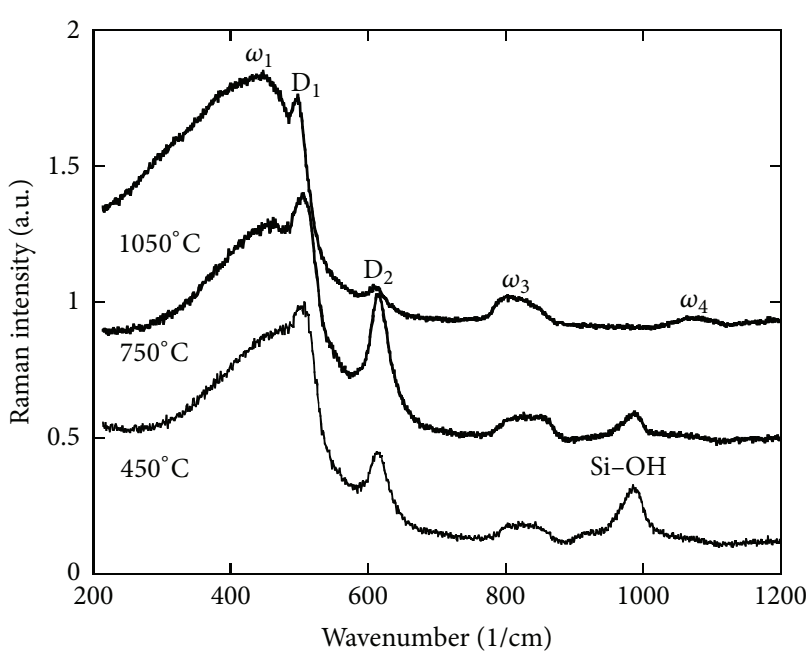

Figure 2: RT Raman spectra of $\mathrm{SiO}_{2}: 0.1 \mathrm{~mol} \%$ Ce samples characterized by different densification temperatures.

heated to $1050^{\circ} \mathrm{C}\left(4^{\circ} \mathrm{C} / \mathrm{h}\right)$ under synthetic air. However, we remark that the use of these different atmospheres in the final densification stage did not significantly influence the investigated properties of the materials. Finally, the oven was switched off and the temperature was decreased to room temperature in about $10 \mathrm{~h}$.

Plates $15 \mathrm{~mm}$ in diameter and $1 \mathrm{~mm}$ thick (sometimes in fragments) were obtained. ICP-MS-LA analysis (Inductively Coupled Plasma-Mass Spectrometer, Perkin Elmer DRC-e, equipped with New Wave UP 213 Laser Ablation sampler) on RE-doped silica glasses confirmed the nominal compositions. Besides, $\mathrm{OH}$ content, monitored by IR absorption, was lower than $1 \mathrm{~mol} \%$.

The influence of the densification process of the xerogels on the structural properties was investigated by Raman spectroscopy. As an example, in Figure 2 the Raman spectra of $\mathrm{SiO}_{2}: 0.1 \mathrm{~mol} \% \mathrm{Ce}$ at different densification temperatures $\left(T_{d}\right)$ are reported: at the lower temperature of $450^{\circ} \mathrm{C}$, intrinsic Raman features of $\mathrm{SiO}_{2}$ are observed at about 440, 800, and $1060 \mathrm{~cm}^{-1}\left(\omega_{1}, \omega_{3}\right.$, and $\omega_{4}$, resp. $)$ [25], together with $\mathrm{D}_{1}$ and $\mathrm{D}_{2}$ peaks at $490 \mathrm{~cm}^{-1}$ and $610 \mathrm{~cm}^{-1}$ assigned to symmetric stretching modes of fourfold and threefold rings of $\mathrm{SiO}_{2}$ tetrahedra [26]; moreover, peaks at 920 and $980 \mathrm{~cm}^{-1}$ and attributed to $\mathrm{Si}-\mathrm{OH}$ stretching modes [27] are detected. Upon increase of $T_{d}$, these peaks decrease and are no more observed after densification at $1050^{\circ} \mathrm{C}$. At the same time, the intensity of the $\omega_{1}$ band increases with respect to $D_{1}$ while the $D_{2}$ peak increases after $750^{\circ} \mathrm{C}$ densification and is lowered after the last $1050^{\circ} \mathrm{C}$ treatment. The Raman spectrum of the $1050^{\circ} \mathrm{C}$ densified silica strongly resembles that of vitreous $\mathrm{SiO}_{2}$ [28], indicating that such treatment leads to a complete glass formation. Moreover, the disappearance of $\mathrm{OH}$-related bands proves that such thermal process provides desorption of the majority of $\mathrm{OH}$ groups initially present in the xerogels, as put in evidence in better detail by IR absorption measurements [29].

On the other hand, in the doping range $0.5-5 \mathrm{~mol} \%$ Ce, the appearance of a Raman peak at $\sim 460 \mathrm{~cm}^{-1}$ was 


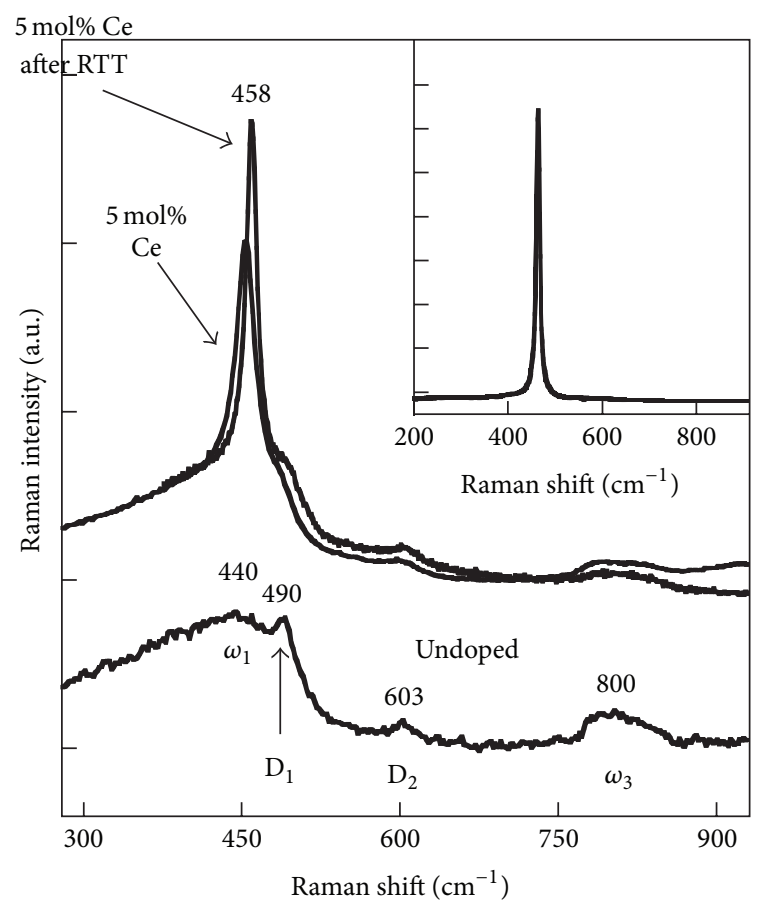

FIgURE 3: Raman spectra obtained on $\mathrm{SiO}_{2}: 5 \mathrm{~mol} \%$ Ce before and after RTT. For comparison the spectrum of undoped $\mathrm{SiO}_{2}$ is also shown. In the inset the Raman spectrum of pure $\mathrm{CeO}_{2}$ raw powder is displayed.

evidenced (Figure 3). This peak could be assigned to a $\mathrm{CeO}_{2}$ crystalline phase after comparison with a commercial powder of pure $\mathrm{CeO}_{2}$, yielding a symmetrical stretching vibration $\left(\mathrm{F}_{2 \mathrm{~g}}\right.$ symmetry of a fluorite structure) at $464 \mathrm{~cm}^{-1}$ [30]. The Full Width at Half Maximum (FWHM) of the peak was observed to decrease by increasing Ce concentration and by RTT, suggesting an increase of the size of $\mathrm{CeO}_{2}$ nanocrystals. Following parallel investigations including also X-ray diffraction (XRD) and transmission electron microscopy (TEM) analyses, the size of such clusters was found to be approximately $15-20 \mathrm{~nm}$ after RTT treatments, slightly increasing by increasing $\mathrm{Ce}^{3+}$ concentration from 1 up to $5 \mathrm{~mol} \%$. The formation of $\mathrm{CeO}_{2}$ clusters, where $\mathrm{Ce}$ is not luminescent, can surely be considered one of the sources of luminescence quenching observed at high Ce concentration, and it constitutes a drawback preventing the use of high $\mathrm{Ce}$ concentrations in the glasses.

Figure 4 shows typical examples of the radioluminescence (RL) spectra of Ce-doped silica glasses and Eu-doped silica glasses, obtained by irradiating the samples with $32 \mathrm{kV}$ X-rays.

The RL spectrum of the Ce-doped silica glass was characterized by a broad emission band, centred around $480 \mathrm{~nm}$, related to the radiative transition $5 \mathrm{~d}-4 \mathrm{f}$ of $\mathrm{Ce}^{3+}$. The $\mathrm{RL}$ spectrum of Eu-doped glass was characterized by a narrow main peak around $620 \mathrm{~nm}$ related to the ${ }^{5} \mathrm{D}_{0}{ }^{-}{ }^{7} \mathrm{~F}_{2}$ transition of $\mathrm{Eu}^{3+}$. Weaker peaks below and above this wavelength due to the further transitions ${ }^{5} \mathrm{D}_{0}-{ }^{7} \mathrm{~F}_{j}(j=1,3,4)$ of $\mathrm{Eu}^{3+}$ can also be observed.

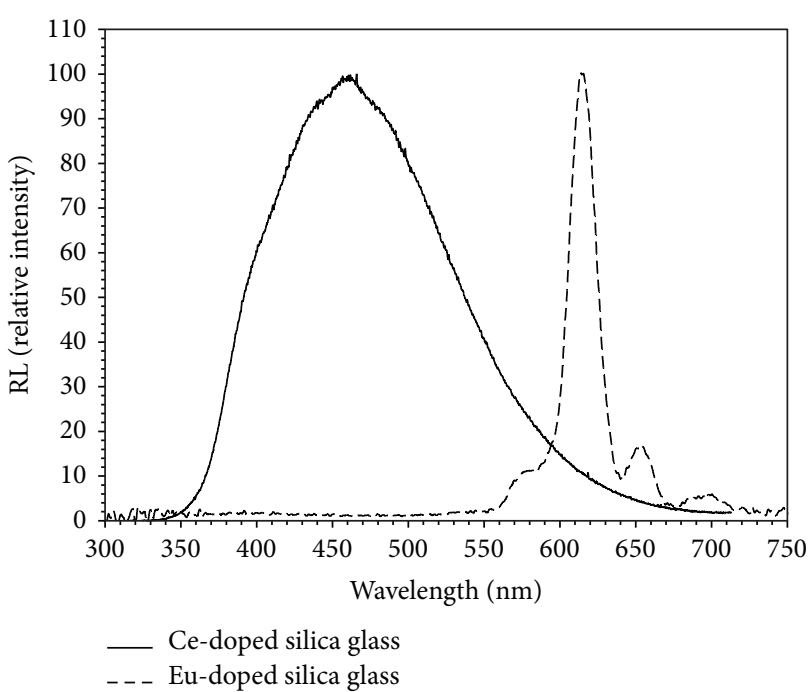

FIGURE 4: RL spectra of Ce- and Eu-doped silica glasses irradiated with $32 \mathrm{kV}$ X-rays.

A rapid thermal treatment (RTT) can be performed after densification at $1050^{\circ} \mathrm{C}$ in order to improve the scintillation properties of the RE by using an oxidizing oxygen-hydrogen flame: after a very quick temperature increase $(2-4 \mathrm{~s})$, the sample was kept at $1800 \pm 50^{\circ} \mathrm{C}$ for approximately $10 \mathrm{~s}$ and then rapidly cooled in air.

Examples of the temperature ramp used for performing the RTT and the related increase of scintillation efficiency observed in Ce-doped silica glasses are shown in Figures 5(a) and 5(b), respectively. For sake of comparison, the scintillation efficiency of a $7 \times 7 \times 1 \mathrm{~mm}^{3}$ plate of $\mathrm{Bi}_{3} \mathrm{Ge}_{4} \mathrm{O}_{12}$ (BGO) single crystal of high quality grown by Bridgman technique at the Shonan Institute of Technology, Fujisawa, Japan, is also shown in Figure 5(b).

\subsection{Preparation and Characterisation of Doped Silica Optical Fibres}

2.2.1. The Powder in Tube Method. RE-doped $\mathrm{SiO}_{2}$ powders were produced by a simple modification of the synthesis procedure described above to obtain bulk doped-samples. Specifically, in order to produce Ce-doped silica optical fibres, the SOL solution is prepared with the following composition: ethanol 99.9\% $18 \mathrm{~mL}$; TEOS $6 \mathrm{~mL}$; $\mathrm{Ce}\left(\mathrm{NO}_{3}\right)_{3} \cdot 6 \mathrm{H}_{2} \mathrm{O}$ solution in ethanol $(10 \mathrm{mg} / \mathrm{mL})$ in the amount to obtain doping of 600 molar ppm Ce in $\mathrm{SiO}_{2}$; water $3.6 \mathrm{~mL}$.

The sol-gel transition is reached after several days in thermostatic chamber at $35^{\circ} \mathrm{C}$. Rapid drying in rotating evaporator allows obtaining xerogel powder; further grinding in agate mortar improves the grain size uniformity.

Slow sintering up to $1100^{\circ} \mathrm{C}$ under both oxidizing $\left(\mathrm{O}_{2}\right)$ atmosphere and vacuum in a quartz chamber gives the final glass powder; in detail, a fist ramp at $10^{\circ} \mathrm{C} / \mathrm{h}$ up to $450^{\circ} \mathrm{C}$ is followed by a stasis of 24 hours; a second ramp at 6 or $10^{\circ} \mathrm{C} / \mathrm{h}$ is used to reach the final temperature of $1100^{\circ} \mathrm{C}$. Following this procedure, the powder is treated in vacuum at higher 


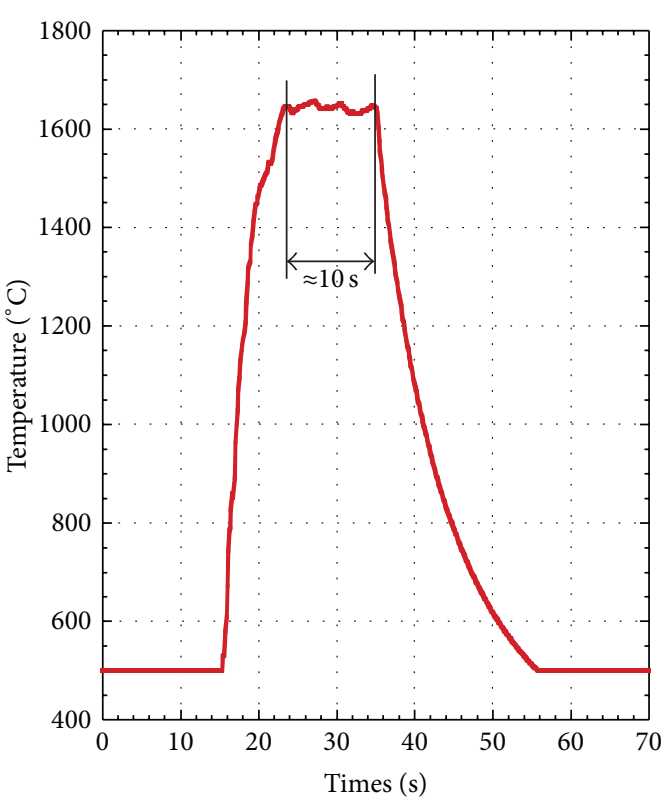

(a)

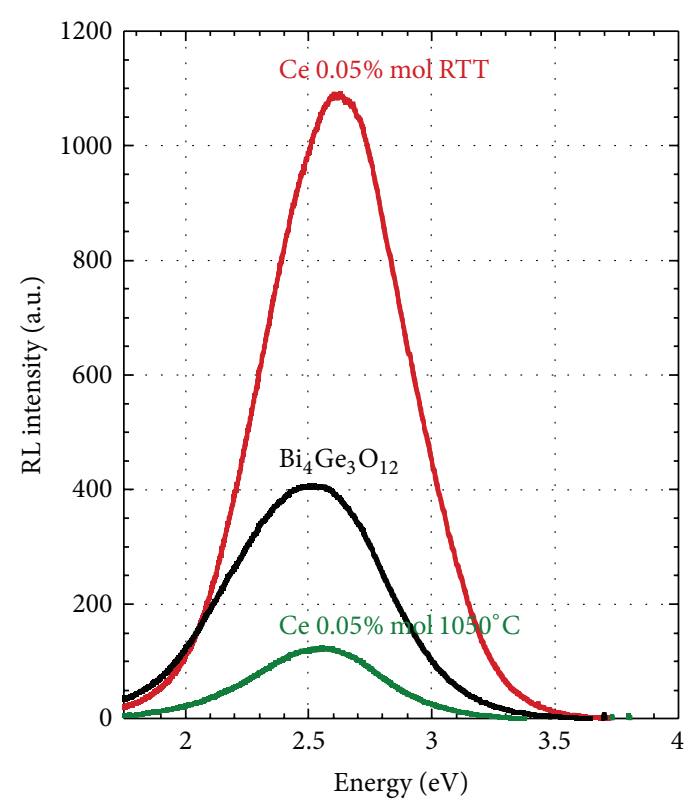

(b)

FIGURE 5: Rapid thermal treatment performed on RE-doped silica glasses: (a) temperature versus time curve used for this procedure (experimental data obtained from optical pyrometer), (b) typical example of scintillation efficiency increase observed in Ce-doped glasses.

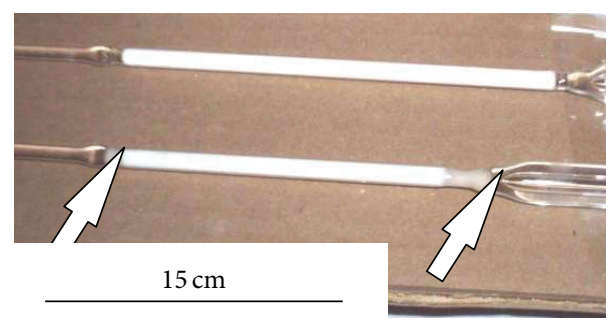

FIGURE 6: Examples of some preforms prepared by the powder in tube method.

temperature $\left(1500-1600^{\circ} \mathrm{C}\right)$ for some minutes (rapid thermal treatment) in order to improve the rare earth scintillation efficiency and remove the $\mathrm{OH}$ content excess. In order to obtain the fibre optic preform, the powder is subsequently introduced in a small suitable quartz tube (Figure 6) under vacuum condition ( $\left.10^{-4}-10^{-5} \mathrm{mbar}\right)$, and voids in the powder are eliminated by using an ultrasonic bath. The tube is vacuum-sealed and then drawn in a furnace at $2100^{\circ} \mathrm{C}$. The fibre diameter has been changed by changing the pulling speed between a minimum of 100 and a maximum of $660 \mu \mathrm{m}$.

The fibre refractive index profile has been analyzed in a fibre with $\sim 105 \mu \mathrm{m}$ diameter using a YORK Instruments S14 analyzer (core dimension $\sim 80 \mu \mathrm{m}$ ). In Figure 7 the refractive index measured along the fibre diameter is reported. The measurement shows that there is no difference between the core and the cladding refractive indexes (the quartz tube container); thus the modification of the silica refractive index induced by the small concentration of dopant is negligible. The guiding effect is given by the silica/air interface that has a numerical aperture $\sim 1$.

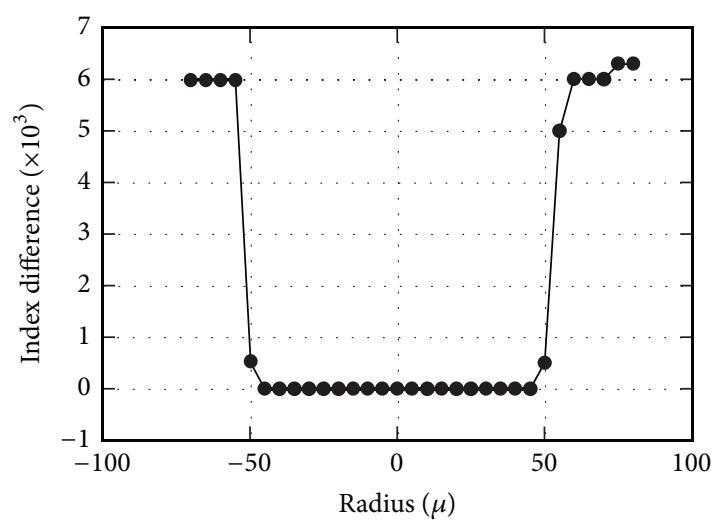

FIgURE 7: Refractive index profile in a fibre with $\sim 105 \mu \mathrm{m}$ total diameter (core diameter equal to $\sim 80 \mu \mathrm{m}$ ).

Due to the nature of the manufacturing process, some voids are not completely closed during the fibre pulling and some sporadic bubbles appear in fibre section (Figure 8). Nevertheless the fraction of scattered light that is lost is small because of the high numerical aperture of the fibre and the degradation of the scintillating signal is limited.

Summarizing, the powder in tube method has proved to have the advantage of a fast and simple preparation; by contrast drawbacks are represented by possible voids due to powder inhomogeneity, imperfect coupling with the cladding, variable diameter, and achievable short fibres (length 2-20 m).

In order to improve the optical and mechanical properties of the doped silica fibres, a new procedure has been optimized, as briefly described in the following section. 


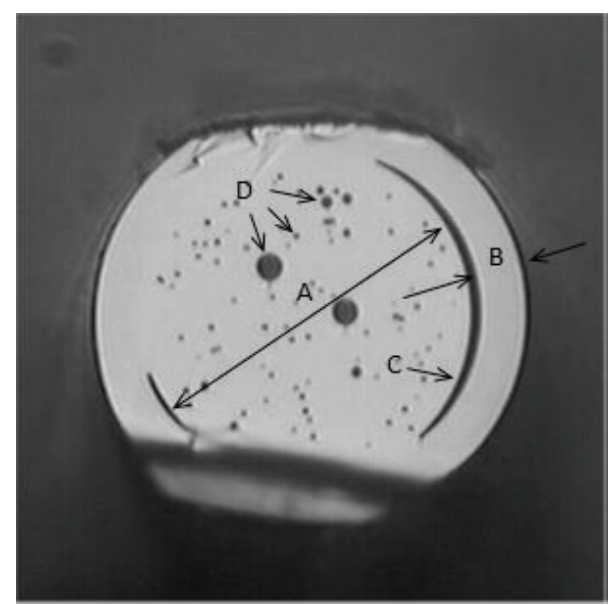

Figure 8: Microscope photograph of a fibre cross-section. The extension of the core $(\mathrm{A})$ and the cladding (B) is clearly visible. Some voids are not completely closed (C) and some bubbles (D) are evident too.

2.2.2. The "Rod in Tube" Method. A similar method to the bulk synthesis described above can be used to produce small cylinders of glass that can be assembled in a suitable preform for optical fibre production. Typical dimensions of such cylinders are 25 to $50 \mathrm{~mm}$ in length and 10 to $15 \mathrm{~mm}$ in diameter. Optical fibres can be obtained without any cladding (or adding a polymeric cladding during pulling) simply by drawing the cylinder welded on a couple of silica handles (Figure 9). Alternatively a fluorine doped silica tube can be collapsed around the RE-doped cylinder to produce a suitable preform resulting in fibres with fluorine doped silica cladding.

The rod in tube method proved to enable a better morphology and homogeneity in the fibre diameter; longer fibres can be obtained (50-500 m), no voids are expected, and totally active fibres can be prepared.

2.3. Doped Silica Optical Fibre Based Dosimeters. Optical fibre based dosimeters were obtained by fusion-splicing (by Starlight Srl, Italy) a $\sim 220 \mu \mathrm{m}$ diameter doped fibre to commercial optical fibres. We used $3 \mathrm{M}$ Hard Clad multimode commercial fibers, with numerical aperture equal to 0.48 , whose silica core was $\sim 200 \mu \mathrm{m}$ in diameter $(\sim 225 \mu \mathrm{m}$ considering the cladding).

Traditionally, cerium was used as dopant because of its very high RL efficiency. Ce-doped silica optical fibres proved to be an effective tool for dosimetric studies carried out with both soft X-rays [31,32] and more energetic fields used in conventional radiotherapy $[33,34]$ and in proton therapy [35].

These studies were performed by using a photomultiplier tube (Hamamatsu, R7400 P) operating in photon-counting mode as optical detector. The signal was then processed by a dedicated acquisition unit (EL.SE s.r.l., Italy) which enabled the selection of different integration times of the signal in the range between $10 \mathrm{~ms}$ and $1 \mathrm{~min}$. Figure 10 shows, for example,

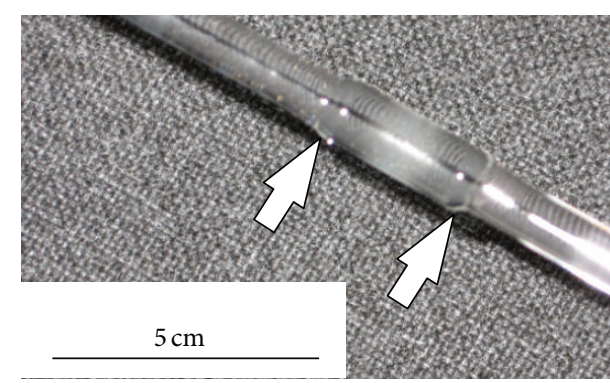

FIGURE 9: Example of a preform prepared by the rod in tube method.

the RL versus time signals obtained by irradiating the Cedoped fibre in two different experimental setups: (a) photons beams produced by a $6 \mathrm{MV}$ linear accelerator operating at different dose rates and (b) protons with an initial energy of $138 \mathrm{MeV}$ produced by a cyclotron using the spot scanning technique.

The high RL efficiency of Ce-doped fibres makes the stem effect contribution almost negligible when stereotactic fields are used [34]. By contrast, the spurious signal needs to be subtracted when the fibre is irradiated with larger fields and/or at different beam orientations. In this situation, only the use of a second reference fibre enabled an effective subtraction of the stem effect: for such reason, the acquisition unit was designed to also contain a secondary PMT, identical to the first one, which enables the measurement of the stem effect produced by the irradiation of the second fibre, parallel to the first one, but without the doped portion.

Other methods for removing the stem effect cannot be applied. Indeed, temporal gating is not usable because of the fast scintillation time of $\mathrm{Ce}^{3+}$ : indeed, the main component of the scintillating time was assessed equal to approximately $55 \mathrm{~ns}$ [31]. Furthermore, chromatic separation is not suitable since the scintillation emission band of $\mathrm{Ce}^{3+}$ totally overlaps the spurious luminescence spectrum, extending in the UVVIS region.

Recently, our research has been addressing the use of dopants emitting in the red and IR region of the visible spectrum in order to implement alternative methods for removing the stem effect contribution on the basis of the analysis of the RL spectra or optical filtering [36-39]. For this purpose, $\mathrm{Eu}$ - and $\mathrm{Yb}$-doped silica fibres were produced and a thermoelectric cooled back-thinned CCD array spectrometer (Prime X, B\&W Tek Inc., USA) operating within a wavelength range $200-990 \mathrm{~nm}$ was used as optical detector.

The narrow main peak around $620 \mathrm{~nm}$ in the RL spectrum of Eu-doped fibre clearly emerged from the continuous background signal due to the stem effect, as shown in Figure 11. The net area of the $\mathrm{Eu}^{3+}$ peak, namely, the RL signal over the spurious signal, proved to act as a Cerenkov-free dosimetric signal.

A validation of this methodology for removing the stem effect was obtained through the physical and dosimetric characterization of $6 \mathrm{MV}$ large photon fields (measurement of the output factors, the depth-dose-profile, and transversal beam profile) and by measuring the RL signals produced by $6 \mathrm{MeV}$ 


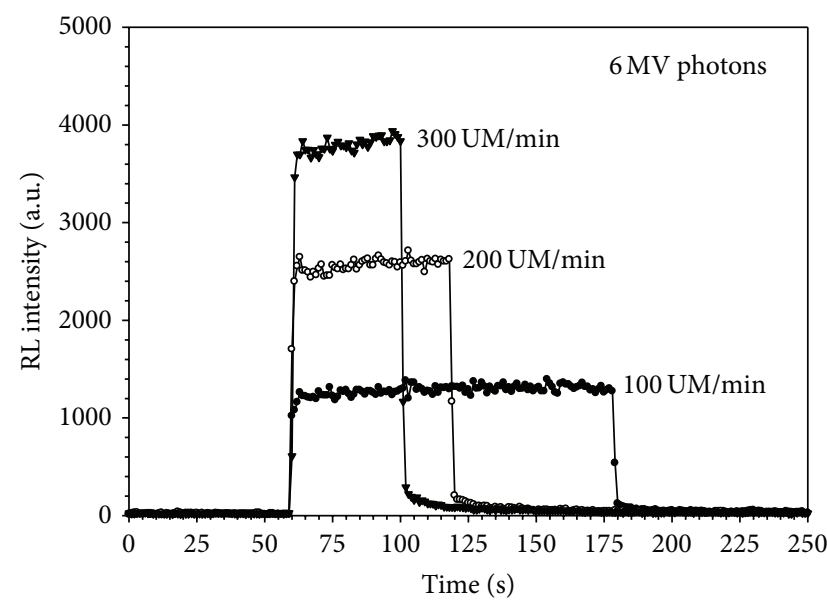

(a)

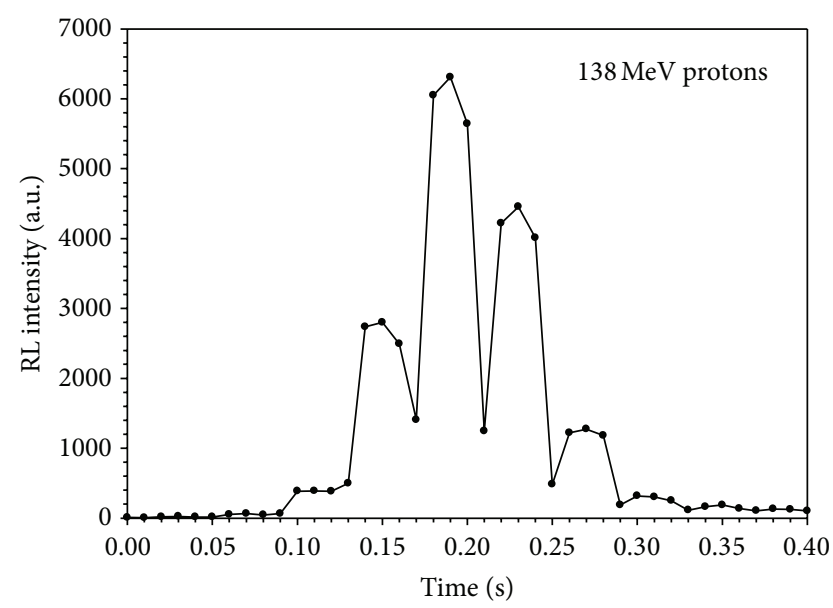

(b)

FIGURE 10: Examples of RL versus irradiation time of the Ce-doped fibre: (a) $6 \mathrm{MV}$ photon beam at different dose rate (integration time 1 second), (b) protons with initial energy of $138 \mathrm{MeV}$ (integration time 10 milliseconds).

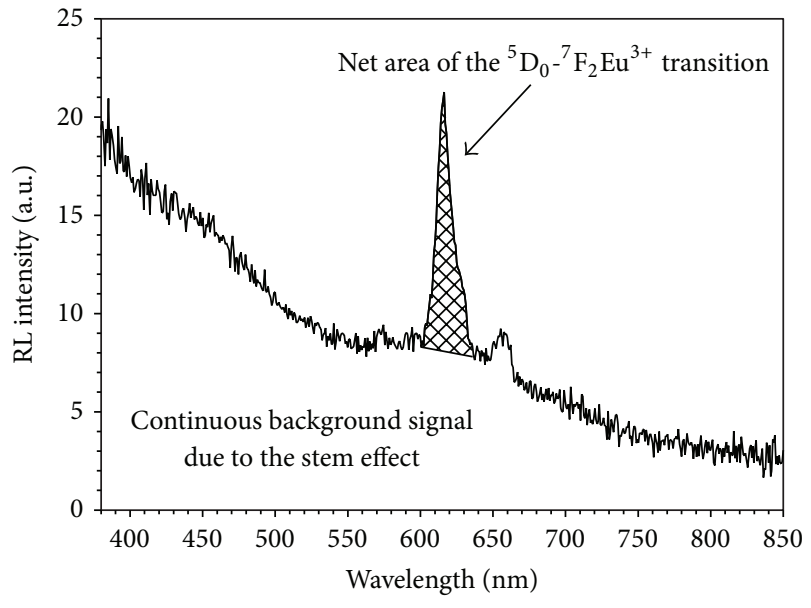

FIGURE 11: The RL spectrum of Eu-doped fibre and the stem effect contribution (irradiation with $6 \mathrm{MV}$ photons beam).

electron beams impinging the doped fibre at different angles $[36,37]$. It must be noted that this method for removing the spurious signal does not require any preliminary calibration, as well as any assumptions about the invariance of the stem effect spectrum.

Finally, the luminescence and dosimetric properties of Yb-doped silica optical fibres are currently being studied. The sharp RL emission line at $975 \mathrm{~nm}$ due to the ${ }^{2} \mathrm{~F}_{5 / 2^{-}}$ ${ }^{2} \mathrm{~F}_{7 / 2}$ transition of $\mathrm{Yb}^{3+}$ makes this dopant very promising for a prompt and efficient subtraction of the stem effect contribution simply by optical filtering [39].

\section{Conclusions}

In conclusion, the reported data have demonstrated that intense luminescence is emitted in rare earth doped silica fibres, whose structural and refractive index properties are very close to those of pure $\mathrm{SiO}_{2}$ glass. Easy coupling can be made between active luminescent fibres and commercial light transmitting fibres to realize a composite fibre for remote dosimetry.

The RL tests performed until now with different radiation sources demonstrated a very good linearity in an extended dose rate interval, suggesting that application could be found in the medical field for both diagnostics and therapy radiation monitoring.

The experiments were performed using different diagnostic radiological equipment: mammography system, conventional X-rays system, and CT scanner but also protons produced by cyclotron.

The obtained results are very satisfactory in sensitivity of the detector, response linearity on a wide range of doses, response independence to environmental conditions (pressure, temperature, and humidity), response stability to short and long time intervals, and response stability and reproducibility after delivering high doses.

In the medical field the new dosimetric system is not alternative to conventional dosimetry systems as ionization chambers, but complementary. This detector, that can be used for radiation beam characterisation, is also particularly promising for in vivo dosimetry. The fibres are safe because they are not electrically powered and are especially indicated for in vivo dosimetry measurements as they assure very high spatial resolution for their geometric features (little diameter and volume) and negligible shading from radiation in comparison to other dosimetric systems.

Feasibility studies for the use of these doped fibres in field different from the medical applications are currently in progress. Particularly, due to the high radiation hardness, such scintillators could find applications in future detectors and calorimeters for high energy physics. A further field of interest could be their application in industrial radiogenic equipment or nuclear reactors in order to evaluate the dose for components that need substitution under intense radiation fields. 


\section{Conflict of Interests}

The authors declare that there is no conflict of interests regarding the publication of this paper.

\section{References}

[1] C. J. Marckmann, M. C. Aznar, C. E. Andersen, and L. BøtterJensen, "Influence of the stem effect on radioluminescence signals from optical fibre $\mathrm{Al}_{2} \mathrm{O}_{3}: \mathrm{C}$ dosemeters," Radiation Protection Dosimetry, vol. 119, no. 1-4, pp. 363-367, 2006.

[2] I. Veronese, M. Fasoli, M. Martini et al., "Phosphorescence of $\mathrm{SiO}_{2}$ optical fibres doped with $\mathrm{Ce}^{3+}$ ions," Physica Status Solidi (C) Current Topics in Solid State Physics, vol. 4, no. 3, pp. 10241027, 2007.

[3] J. V. Jelley, Cerenkov Radiation and Its Applications, Pergamon Press, 1958.

[4] A. S. Beddar, T. R. Mackie, and F. H. Attix, "Cerenkov light generated in optical fibres and other light pipes irradiated by electron beams," Physics in Medicine and Biology, vol. 37, no. 4, pp. 925-935, 1992.

[5] A. S. Beddar, T. R. Mckie, and F. H. Attix, "Water-equivalent plastic scintillation detectors for high-energy beam dosimetry: I. Physical characteristics and theoretical considerations," Physics in Medicine and Biology, vol. 37, no. 10, pp. 1883-1900, 1992.

[6] S. F. de Boer, A. S. Beddar, and J. A. Rawlinson, "Optical filtering and spectral measurements of radiation-induced light in plastic scintillation dosimetry," Physics in Medicine and Biology, vol. 38, no. 7, pp. 945-958, 1993.

[7] D. Flühs, M. Heintz, F. Indenkämpen, C. Wieczorek, H. Kolanoski, and U. Quast, "Direct reading measurement of absorbed dose with plastic scintillators-the general concept and applications to ophthalmic plaque dosimetry," Medical Physics, vol. 23, no. 3, pp. 427-434, 1996.

[8] D. Létourneau, J. Pouliot, and R. Roy, "Miniature scintillating detector for small field radiation therapy," Medical Physics, vol. 26, no. 12, pp. 2555-2561, 1999.

[9] S. Broggi, M. C. Cantone, A. Chiara et al., "Application of failure mode and effects analysis (FMEA) to pretreatment phases in tomotherapy," Journal of Applied Clinical Medical Physics, vol. 14, no. 5, pp. 265-277, 2013.

[10] M. C. Cantone, M. Ciocca, F. Dionisi et al., "Application of failure mode and effects analysis to treatment planning in scanned proton beam radiotherapy," Radiation Oncology, vol. 8, no. 1, article 127, 2013.

[11] M. Ciocca, M.-C. Cantone, I. Veronese et al., "Application of failure mode and effects analysis to intraoperative radiation therapy using mobile electron linear accelerators," International Journal of Radiation Oncology Biology Physics, vol. 82, no. 2, pp. e305-e311, 2012.

[12] P. Z. Y. Liu, N. Suchowerska, J. Lambert, P. Abolfathi, and D. R. McKenzie, "Plastic scintillation dosimetry: comparison of three solutions for the Cerenkov challenge," Physics in Medicine and Biology, vol. 56, no. 18, pp. 5805-5821, 2011.

[13] L. Archambault, L. Beaulieu, and S. A. Beddar, "Comment on 'plastic scintillation dosimetry: comparison of three solutions for the Cerenkov challenge," Physics in Medicine and Biology, vol. 57, no. 11, pp. 3661-3665, 2012.

[14] M. A. Clift, P. N. Johnston, and D. V. Webb, "A temporal method of avoiding the Cerenkov radiation generated in organic scintillator dosimeters by pulsed mega-voltage electron and photon beams," Physics in Medicine and Biology, vol. 47, no. 8, pp. 14211433, 2002.

[15] C. E. Andersen, S. M. S. Damkjær, G. Kertzscher, S. Greilich, and M. C. Aznar, "Fiber-coupled radioluminescence dosimetry with saturated $\mathrm{Al}_{2} \mathrm{O}_{3}: \mathrm{C}$ crystals: characterization in 6 and 18 MV photon beams," Radiation Measurements, vol. 46, no. 10, pp. 1090-1098, 2011.

[16] J. M. Fontbonne, G. Iltis, G. Ban et al., "Scintillating fiber dosimeter for radiation therapy accelerator," IEEE Transactions on Nuclear Science, vol. 49, no. 5, pp. 2223-2227, 2002.

[17] A.-M. Frelin, J.-M. Fontbonne, G. Ban et al., "Spectral discrimination of Čerenkov radiation in scintillating dosimeters," Medical Physics, vol. 32, no. 9, pp. 3000-3006, 2005.

[18] M. Guillot, L. Gingras, L. Archambault, S. Beddar, and L. Beaulieu, "Spectral method for the correction of the Cerenkov light effect in plastic scintillation detectors: a comparison study of calibration procedures and validation in Cerenkov lightdominated situations," Medical Physics, vol. 38, no. 4, pp. 21402150, 2011.

[19] J. Lambert, Y. Yin, D. R. McKenzie, S. Law, and N. Suchowerska, "Cerenkov-free scintillation dosimetry in external beam radiotherapy with an air core light guide," Physics in Medicine and Biology, vol. 53, no. 11, pp. 3071-3080, 2008.

[20] J. Lambert, D. R. McKenzie, S. Law, J. Elsey, and N. Suchowerska, "A plastic scintillation dosimeter for high dose rate brachytherapy," Physics in Medicine and Biology, vol. 51, no. 21, pp. 5505-5516, 2006.

[21] M. Carrara, C. Cavatorta, M. Borroni et al., "Characterization of a $\mathrm{Ce}^{3+}$ doped $\mathrm{SiO}_{2}$ optical dosimeter for dose measurements in HDR brachytherapy," Radiation Measurements, vol. 56, pp. 312-315, 2013.

[22] M. Carrara, C. Tenconi, R. Guilizzoni et al., "Stem effect of a $\mathrm{Ce}^{3+}$ doped $\mathrm{SiO}_{2}$ optical dosimeter irradiated with a ${ }^{192}$ Ir HDR brachytherapy source," Radiation Physics and Chemistry, vol. 104, pp. 175-179, 2014.

[23] S. Buranurak, C. E. Andersen, A. R. Beierholm, and L. R. Lindvold, "Temperature variations as a source of uncertainty in medical fiber-coupled organic plastic scintillator dosimetry," Radiation Measurements, vol. 56, pp. 307-311, 2013.

[24] G. Kertzscher, C. E. Andersen, J. M. Edmund, and K. Tanderup, "Stem signal suppression in fiber-coupled $\mathrm{Al}_{2} \mathrm{O}_{3}: \mathrm{C}$ dosimetry for ${ }^{192}$ Ir brachytherapy," Radiation Measurements, vol. 46, no. 12, pp. 2020-2024, 2011.

[25] F. L. Galeener, G. Lucovsky, and R. H. Geils, "Raman and infrared spectra of vitreous $\mathrm{As}_{2} \mathrm{O}_{3}$," Physical Review B, vol. 19, no. 8 , pp. 4251-4258, 1979.

[26] A. Pasquarello and R. Car, "Identification of Raman defect lines as signatures of ring structures in vitreous silica," Physical Review Letters, vol. 80, no. 23, pp. 5145-5147, 1998.

[27] C. A. Murray and T. J. Greytak, "Intrinsic surface phonons in amorphous silica," Physical Review B, vol. 20, no. 8, pp. 33683387, 1979.

[28] S. K. Sharma, J. F. Mammone, and M. F. Nicol, "Raman investigation of ring configurations in vitreous silica," Nature, vol. 292, no. 5819, pp. 140-141, 1981.

[29] A. Baraldi, R. Capelletti, N. Chiodini et al., "Vibrational spectroscopy of $\mathrm{OH}$-related groups in $\mathrm{Ce}^{3+}$ - and $\mathrm{Gd}^{3+}$-doped silicate glasses," Nuclear Instruments and Methods in Physics Research Section A, vol. 486, no. 1-2, pp. 408-411, 2002.

[30] W. H. Weber, K. C. Hass, and J. R. McBride, "Raman study of $\mathrm{CeO}_{2}$ : second-order scattering, lattice dynamics, and particlesize effects," Physical Review B, vol. 48, article 178, 1993. 
[31] A. Vedda, N. Chiodini, D. Di Martino et al., "Ce $\mathrm{C}^{3+}$-doped fibers for remote radiation dosimetry," Applied Physics Letters, vol. 85, no. 26, pp. 6356-6358, 2004.

[32] N. Caretto, N. Chiodini, F. Moretti, D. Origgi, G. Tosi, and A. Vedda, "Feasibility of dose assessment in radiological diagnostic equipments using Ce-doped radio-luminescent optical fibers," Nuclear Instruments and Methods in Physics Research A: Accelerators, Spectrometers, Detectors and Associated Equipment, vol. 612, no. 2, pp. 407-411, 2010.

[33] E. Mones, I. Veronese, F. Moretti et al., "Feasibility study for the use of $\mathrm{Ce}^{3+}$-doped optical fibres in radiotherapy," Nuclear Instruments and Methods in Physics Research, Section A: Accelerators, Spectrometers, Detectors and Associated Equipment, vol. 562, no. 1, pp. 449-455, 2006.

[34] E. Mones, I. Veronese, A. Vedda et al., "Ce-doped optical fibre as radioluminescent dosimeter in radiotherapy," Radiation Measurements, vol. 43, no. 2-6, pp. 888-892, 2008.

[35] I. Veronese, M. C. Cantone, N. Chiodini et al., "Feasibility study for the use of cerium-doped silica fibres in proton therapy," Radiation Measurements, vol. 45, no. 3-6, pp. 635-639, 2010.

[36] I. Veronese, M. C. Cantone, M. Catalano et al., "Study of the radioluminesence spectra of doped silica optical fibre dosimeters for stem effect removal," Journal of Physics D: Applied Physics, vol. 46, no. 1, Article ID 015101, 2013.

[37] I. Veronese, M. C. Cantone, N. Chiodini et al., "The influence of the stem effect in Eu-doped silica optical fibres," Radiation Measurements, vol. 56, pp. 316-319, 2013.

[38] I. Veronese, M. C. Cantone, N. Chiodini et al., "Radioluminescence dosimetry by scintillating fiber optics: the open challenges," in Hard X-Ray, Gamma-Ray and Neutron Detector Physics XV, vol. 8852 of Proceedings of SPIE, 88521L, August 2013.

[39] I. Veronese, C. de Mattia, M. Fasoli et al., "Infrared luminescence for real time ionizing radiation detection," Applied Physics Letters, vol. 105, no. 6, Article ID 061103, 2014. 

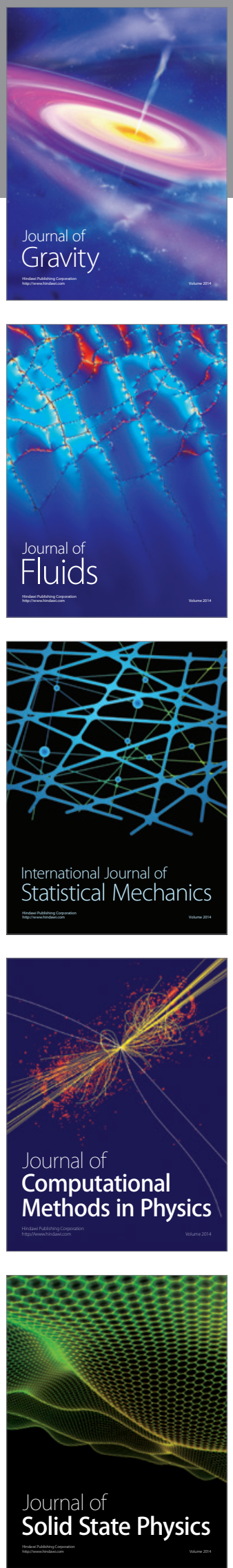

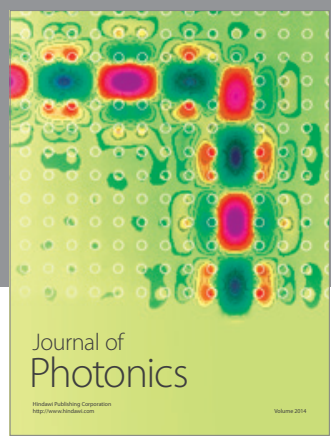

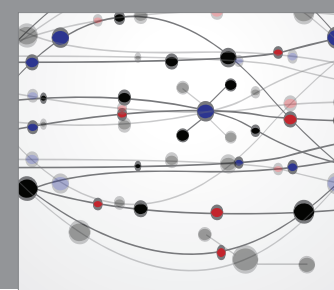

The Scientific World Journal

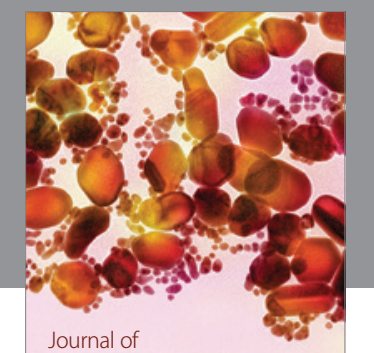

Soft Matter
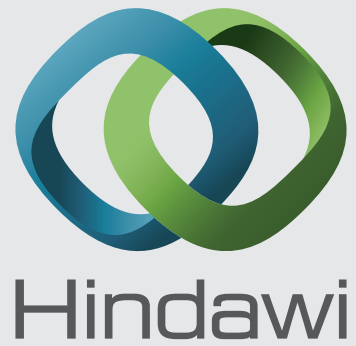

Submit your manuscripts at

http://www.hindawi.com
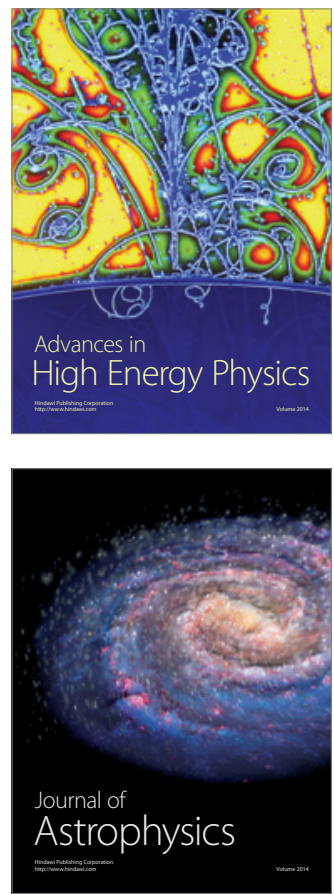
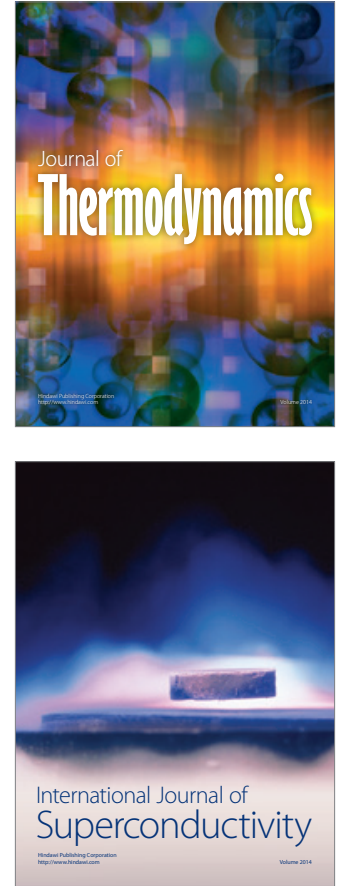
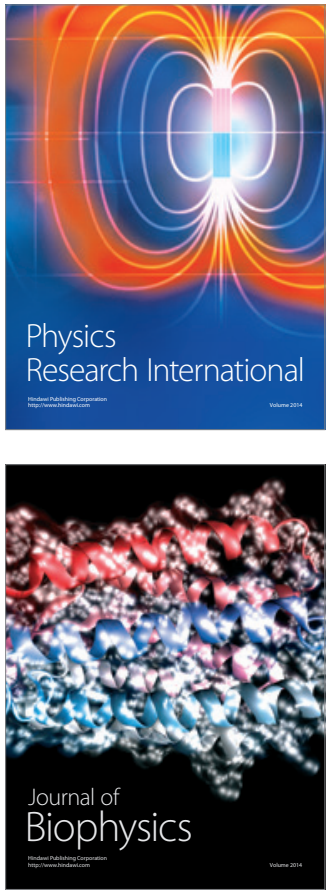
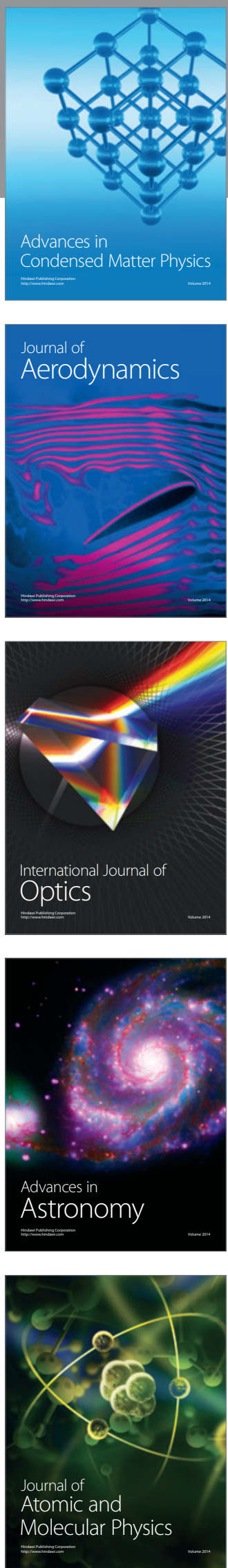